\title{
Discrimination of second-formant-like frequency transitions
}

\author{
LOIS L. ELLIOTT, MICHAEL A. HAMMER, and THOMAS CARRELL \\ Northwestern University, Evanston, Illinois
}

\begin{abstract}
Discrimination of two types of simulated single-second-formant frequency transitions was studied, transitions where members of a stimulus set shared the same onset frequency and transitions where stimuli of a set shared the same offset frequency. Experiment 1 employed an adaptive procedure to measure just-noticeable differences for transitions that increased in frequency. Experiment 2 obtained complete psychometric functions, based on a three-interval, forced-choice procedure, for transitions that either increased or decreased in frequency. In both experiments, better discrimination occurred for longer stimuli, measured in hertz/millisecond, and for transitions that shared the same onset frequency. There were no differences, in Experiment 2, between discrimination of rising and falling transitions. Results are considered from several theoretical perspectives.
\end{abstract}

In recent decades there has been increased interest in studying auditory perception of frequency transitions (glissandi, or frequency glides) (e.g., Pols \& Schouten, 1987; Schouten, 1985). Much of this research has been motivated by the fact that many speech consonants contain frequency transitions that must be perceived in order for the consonant to be identified. This work has variously required the listener to determine whether a tone changes to a higher or lower frequency (e.g., Pollack, 1968; Sergeant \& Harris, 1962), to detect a frequency transition (e.g., Arlinger, Jerlvall, Ahren, \& Holmgren, 1977; Gardner \& Wilson, 1979), or to judge two tonal glides as "same" or "different" (e.g., Nabelek \& Hirsh, 1969).

Discrimination within sets of transitions with the same offset frequency is somewhat analogous to the listener's task in discriminating between certain word-initial stop consonants that precede the same vowel. In this instance, the second and third formants of the different consonants would begin at different frequencies but converge to the same vowel formants. Similarly, differentiation between certain stop consonants that follow the same vowel requires discrimination of formant transitions that begin at the same frequency but diverge to different frequencies. A major purpose of this experiment was to examine the discrimination of transition stimuli that simulated these second-formant-like circumstances.

This approach differed from the work of Schouten and Pols (1989), who studied discrimination between rising and steady-state stimuli, between falling and steady-state

This research was supported, in part, by a grant from NIDCD (NIH). Experiment 1 was reported at the meeting of the Association for Research in Otolaryngology, February 1990. We thank Jan Wasowicz, Bob Loyd, Rich Benassi, and the late Jim Baker for technical assistance. We also thank two anonymous reviewers for their comments on a previous version of this paper. Correspondence may be addressed to Lois L. Elliott, Department of Communication Sciences and Disorders, Northwestern University, 2299 Sheridan Road, Evanston, IL 60208-3550. stimuli, and between rising and falling stimuli. In contrast, our experiments required discrimination between transitions that all were rising or between transitions that all were falling. We defined transition-discrimination as the smallest differences between members of a stimulus set that could be responded to correctly $50 \%$ of the time (Experiments 1 and 2) or 70\% of the time (Experiment 2).

Our work also differed from the third test condition of a study by Tyler, Wood, and Fernandes (1983), who used rising 50-msec transitions that ended at the same frequency. Our experiment examined discrimination between transitions that increased or that decreased in frequency at different rates. Furthermore, in this study, transitions to be discriminated either began or ended at the same frequency.

Nabelek, Nabelek, and Hirsh (1970) reported that, as stimulus duration increased to $120 \mathrm{msec}$, the perceived pitch of linearly changing tone bursts more closely matched the final frequency than did that of shorter tone bursts. This outcome suggested Hypothesis 1, that better discrimination should occur for transitions that diverge from a common frequency than for transitions that converge, assuming that perceived pitch of band sweeps follows the same patterns as Nabelek et al.'s tonal stimuli. This would happen because converging transitions would be perceived as having more similar pitches than would diverging transitions.

Schouten and Pols (1989) concluded that "sweep perception is determined mainly by the difference between initial and final frequency" (p. 244). It might be proposed that this conclusion may be related to a sampling theory of transition discrimination, which was defined by Dooley and Moore (1988) as based on perceiving "differences in frequency between samples of the stimulus taken at different points in time"' (p. 2045). This interpretation would lead to several additional hypotheses: (2) Among sets of transitions that change at the same rate, discrimination of longer transitions should be better than discrimination of shorter transitions because the longer stimuli 
sweep through larger frequency differences and would allow opportunities for taking more samples. (3) There should be no discrimination differences between rising and falling transitions if both sweep through the same frequency extents. Much previous work supported this last hypothesis (e.g., Arlinger et al., 1977; Collins, 1984; Dooley \& Moore, 1988, Experiment 2; Elliott, Hammer, Scholl, Carrell, \& Wasowicz, 1989; Nabelek \& Hirsh, 1969). However, some investigators have reported differences attributable to transition direction (e.g., Carlyon \& Stubbs, 1989; Collins, Cullen, Porter, \& Jackson, 1988).

Dooley and Moore (1988) and Moore and Glasberg (1989) considered whether Zwicker's (1970) excitationpattern model of frequency discrimination was applicable to frequency glides, and found some (but not complete) support for this model. Zwicker's model assumes that the listener attends to the low-frequency side of the excitation pattern. For rising transitions, then, it would seem reasonable to predict better discrimination for a set of transitions in which individual members begin at different frequencies and rise to converge to the same point than for a set in which transitions rise and diverge. In the former case, the low-frequency portions of the stimuli within a set all differ, whereas in the latter case they are all identical. This prediction, for rising transitions, is opposite to Hypothesis 1 (which predicts better discrimination for transitions that diverge from a common frequency than for transitions that converge). In contrast, for falling transitions, the excitation pattern model would seem to predict better discrimination among the set of stimuli that fall and diverge in frequency than among stimuli that fall and converge. The latter prediction is in accordance with Hypothesis 1.

Two experiments were conducted. In Experiment 1, justnoticeable differences (JNDs) were obtained using an adaptive procedure; in Experiment 2, complete psychophysical functions were obtained. In Experiment 1, only sets of stimuli with rising frequencies were used; in Experiment 2 , sets of stimuli with both rising and falling fre- quencies were used. Thus, Experiment 1 tested the first two hypotheses, ${ }^{1}$ while Experiment 2 tested all of them.

\section{STIMULI FOR BOTH EXPERIMENTS}

Eight sets of stimuli (filtered series of impulses) were generated with the Klatt $(1980,1987)$ parallel/cascade formant synthesizer using all-parallel synthesis. Center frequencies of the filter at onset and offset were selected, on the basis of pilot testing, to ensure that discrimination could be measured without encountering "floor" or "ceiling" effects. That is, listeners with normal hearing were expected to discriminate among at least some members of a set and no listener could consistently discriminate among adjacent stimuli of a set. Four different types of stimuli were used, two that increased linearly in frequency and two that decreased in frequency. For the stimulus sets that increased in frequency (Uxx; "U" = "up'), the members of each set either shared the same frequency onset (USO) but had different offsets or had different frequency onsets but shared the same frequency offset (USF). Similar conditions applied to stimulus sets that decreased in frequency ( $\mathrm{Dxx}$; " $\mathrm{D}$ " = "down"). Two durations (60- and 120-msec) were used for each stimulus type, leading to eight stimulus sets. There were 13 stimulus members of each stimulus set. Table 1 displays the center frequency characteristics of each stimulus set.

All transitions were generated by passing a complex periodic source, consisting of a series of filtered impulses with a constant fundamental frequency of $120 \mathrm{~Hz}$, through a single software bandpass filter with an $80-\mathrm{Hz}$ bandwidth. The source spectrum had a 12-dB drop per octave across the harmonics of the FO. The complex periodic source was increased from $0 \mathrm{~dB}$ to maximum amplitude during the initial $10 \mathrm{msec}$ of the stimulus. Thereafter, it was adjusted at 5-msec intervals so that the amplitude of the filter output remained constant as the center frequency of the bandpass filter changed. This amplitude adjustment was accomplished in a two-stage procedure. That is, each transition was synthesized; its amplitude was measured at 5-msec intervals; synthesis parameters were modified to equate output amplitudes; and, finally, the transition was resynthesized with the new, corrected amplitude parameters. ${ }^{2}$ Because transitions were all located in the $1-1.3 \mathrm{kHz}$ region, equating levels was expected to eliminate any possible loudness-difference cues.

A brief, 30-msec noise, equivalent in bandwidth to about four critical bands, was appended to each stimulus. The purpose of this noise was to provide a brief ending for XSO stimulus sets that was identical across all members of each set. In other words, if sub-

Table 1

Center Frequency Characteristics of the 80-Hz Bandpass Filter That Was Used to Generate Stimuli: Beginning and End of Each Stimulus Set

\begin{tabular}{|c|c|c|c|c|c|c|c|}
\hline \multirow{3}{*}{$\begin{array}{c}\text { Stimulus } \\
\text { Type }\end{array}$} & \multirow{3}{*}{$\begin{array}{l}\text { Stimulus } \\
\text { Number }\end{array}$} & \multicolumn{4}{|c|}{ Duration (msec) } & \multirow{2}{*}{\multicolumn{2}{|c|}{$\begin{array}{c}\text { Step Size }(\mathrm{Hz}) \\
\text { Duration (msec) }\end{array}$}} \\
\hline & & \multicolumn{2}{|c|}{60} & \multicolumn{2}{|c|}{120} & & \\
\hline & & Beginning & End & Beginning & End & 60 & 120 \\
\hline \multirow[t]{2}{*}{ USO } & 1 & 1000 & 1012 & 1000 & 1024 & 12 & 24 \\
\hline & 13 & 1000 & 1156 & 1000 & 1312 & & \\
\hline \multirow[t]{2}{*}{ USF } & 1 & 1300 & 1312 & 1288 & 1312 & 12 & 24 \\
\hline & 13 & 1156 & 1312 & 1000 & 1312 & & \\
\hline \multirow[t]{2}{*}{ DSO } & 1 & 1312 & 1300 & 1312 & 1288 & 12 & 24 \\
\hline & 13 & 1312 & 1156 & 1312 & 1000 & & \\
\hline \multirow[t]{2}{*}{ DSF } & 1 & 1012 & 1000 & 1024 & 1000 & 12 & 24 \\
\hline & 13 & 1156 & 1000 & 1312 & 1000 & & \\
\hline
\end{tabular}

Note-XSO, stimuli that shared the same onset frequency; XSF, stimuli that shared the same offset frequency. $X=U$, stimuli that increased in frequency; $X=D$, stimuli that decreased in frequency. * Difference in hertz between center frequencies of the bandpass filter for adjacent members of a stimulus set: at stimulus offset for XSO sets and at stimulus onset for XSF sets. 
jects based their responses only on the final 30-msec portion of the stimulus, which was the brief noise, discrimination of XSF stimuli would be expected to be similar to discrimination of the XSO stimuli-and Hypothesis 1 would not be supported. Because the noise was brief and no more intense than the transition, it was not expected to exert a backward-masking effect. Also, Elliott et al. (1989) found no backward-masking effect when a similar, longer $(180-, 240-$, or $270-\mathrm{msec})$ noise followed the transition.

The noise was increased from $0 \mathrm{~dB}$ to maximum amplitude during the 10 -msec period following the transition at the same time as the amplitude of the complex periodic source was decreased from its maximum amplitude to $0 \mathrm{~dB}$. The center frequency of the bandpass filter used to generate the 30 -msec wide-band noise at the end of stimuli was the same for all stimuli of a set. It was $1000 \mathrm{~Hz}$ for the DSF stimulus set, $1144 \mathrm{~Hz}$ for the DSO set, $1312 \mathrm{~Hz}$ for USF stimuli, and $1336 \mathrm{~Hz}$ for USO stimuli. ${ }^{3}$

The digital signals created with the synthesizer (12-bit resolution; 10K sampling rate) were stored digitally for subsequent testing. None of the stimuli sounded speechlike.

\section{EXPERIMENT 1}

\section{Method}

Stimuli. Stimulus types USO and USF (Table 1) were used. Both 60- and 120-msec stimuli were employed.

Subjects. Ten young adults -5 males and 5 females - with normal hearing participated as subjects. Four had previously listened to synthesized acoustic stimuli; 6 had not. All had learned English as their first language (a point important only because of the resemblance of the stimuli to second formants of some English consonant sounds).

Procedure. An adaptive, simple up-down (Levitt, 1971), computer-controlled procedure was used to test subjects individually. Two stimuli were presented on each trial with a 500 -msec interstimulus interval. The subjects judged each pair of stimuli as "same" or "different." Listeners had unlimited time to respond and received trial-by-trial feedback. Stimuli were presented monaurally via TDH49 headphones at intensities equivalent to approximately 40-dB sensation level.

JNDs were always measured relative to the transition, within a stimulus set, that had the smallest frequency excursion. This stimulus was termed the "anchor" and is identified in Table 1 as Stimulus 1 . The first trial of a test run always presented two stimuli with maximum acoustic differences. Thereafter, the acoustic difference between the anchor and the comparison stimulus of a test trial (i.e., a trial in which the stimuli differed) was decreased after every "different" response and increased after every "same" response. The first two reversals of the adaptive test procedure for each test run were discarded, and the following eight response reversals were used in calculating the JND. The difference between the anchor stimulus and the mean of the stimuli at the reversal points was designated as the JND. Listeners completed six test runs for each stimulus condition. Total listening time for each participant was approximately $2 \mathrm{~h}$, divided into two sessions.

Catch trials, in which the stimuli were identical and the correct response was "same," were employed to prevent the listener from developing a bias towards responding "different." (Had catch trials not been employed, the listener might have learned to respond "different" on every trial-and would have been reinforced for this response.) Stimuli 1 and 1,2 and 2, or 3 and 3 were used for catch trials. These stimuli were intermixed among test trials of a test run and were inserted in a pseudorandom pattern in order to have approximately equal numbers of test and catch trials. Responses to catch-trial stimuli were tabulated separately and did not influence the adaptive test procedure or calculation of the JND.

Listeners were instructed about the task and told that it was impossible to be correct on every trial. They were given several prac-

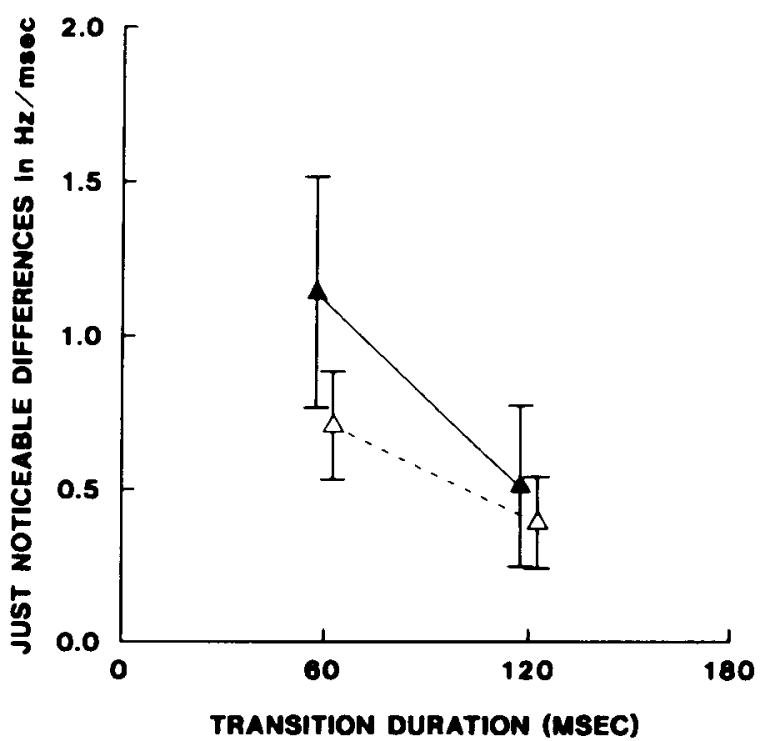

Figure 1. Mean just-noticeable difierences (JND) in hertz/milllsecond for Experiment 1. Symbols: $\triangle$, USO, rising trandtions sharing the same frequency onset; $\Delta$, USF, rising trandtions sharing the same frequency offset. Vertical bars represent $\pm 1 S D$.

tice runs and asked whether they had questions before data collection began.

\section{Results}

Tests of significance revealed no performance differences between subjects who had previously listened to synthesizer-produced acoustic stimuli and those who had not. Accordingly, data for all 10 listeners were pooled over all test runs for each condition. JNDs were evaluated in hertz/millisecond.

Results for the USF and USO (rising) conditions are shown in Figure 1. A two-factor, repeated measures analysis of variance (ANOVA) revealed significantly better discrimination for the USO condition $[F(1,9)=17.54$, $p<.01$ ], supporting Hypothesis 1 . Better discrimination also occurred for longer transitions $[F(1,9)=152.34$, $p<.001]$, supporting Hypothesis 2 .

Catch-trial performance. Mean catch-trial performance is shown in Figure 2. Better performance occurred for longer stimuli $[F(1,9)=17.41, p<.01]$ and for transitions that shared the same onset frequency $[F(1,9)=$ 8.59, $p<.05]$. Catch-trial performance, therefore, generally corresponded to JND performance. (Smaller JNDs and higher percent-correct scores on catch trials represented better performance.)

\section{EXPERIMENT 2}

\section{Method}

Stimuli. The four stimulus sets with rising transitions used in Experiment 1 were employed, along with the four companion sets with falling transitions. 


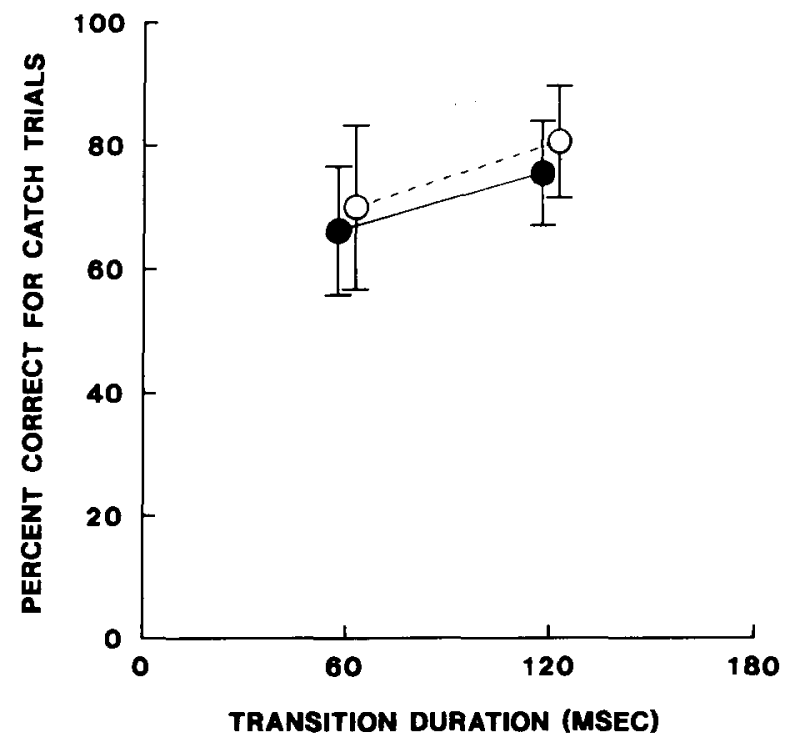

Figure 2. Mean performance, in percent correct, for trials where the correct answer was "same" - that is, for "catch trials." Symbols: O, USO; 9 , USF.

Subjects. Four young adult female subjects with normal hearing participated. All had previously listened to synthesized acoustic stimuli, and all had learned English as their first language. None had participated in Experiment 1.

Procedure. A three-interval, forced-choice (3IFC) psychophysical procedure was used. Listeners pushed one of three buttons to indicate which one of the three stimuli of each trial differed from the other two. The comparison was always made relative to the "anchor" stimulus (which is indicated as Stimulus 1 in Table 1). On any trial, the anchor stimulus could be presented twice and the comparison stimulus once or the comparison stimulus could be presented twice and the anchor once. All 12 members of the stimulus set, other than the anchor, were employed (and selected randomly by the computercontrolled procedure) in order to generate the complete psychophysical function. During a block of trials, each of the 12 nonanchor members of a stimulus set was compared with the anchor 18 times. (In 6 instances, the different stimulus occurred in Interval 1; in another 6 instances, it occurred in Interval 2, etc.) Thus, a block consisted of 216 trials. The three stimuli per trial were separated by $500 \mathrm{msec}$, and the listener had unlimited time to respond. Visual trial-by-trial feedback was provided.

Listeners completed one block of each of the eight stimulus conditions as practice. They then listened to five blocks of each condition, completing a series of all stimulus conditions before beginning the next "round." Different randomizations of conditions were assigned to different listeners. Listening extended over multiple sessions. Total listening time for each participant ranged from 14 to $18 \mathrm{~h}$. All other listening conditions were the same as in Experiment 1 .

\section{Results}

The program that controlled Experiment 2 fit a logistic, psychometric function to the data for each block and determined the values (in stimulus step positions) that were discriminated from the anchor with $50 \%$ and $70 \%$ accuracy. These values were averaged and converted to hertz/ millisecond.

Figure 3, for rising transitions, and Figure 4, for falling transitions, display the mean $70 \%$ correct values in hertz/ millisecond derived from the psychometric functions. For both 60 - and 120 -msec transitions, performance was better for stimulus sets in which transitions shared the same onset frequency than for those in which they shared the same offset frequency $[F(1,3)=10.38, p<.05]$. The threefactor, repeated measures ANOVA also revealed significantly better discrimination for the longer transitions $[F(1,3)=61.78, p<.01]$, thus confirming Hypothesis 2 . Both of these outcomes confirmed results of Experiment 1 and the work of some other investigators on which the hypotheses were originally based (see previous discussion

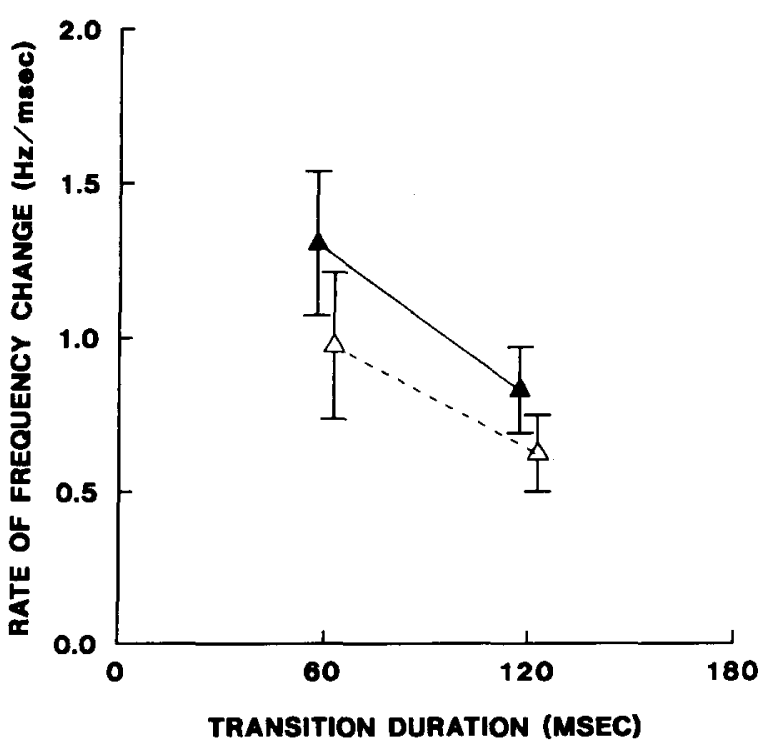

Figure 3. Mean $70 \%$ correct values in hertz/millisecond for rising transitions, Experiment 2. Symbols same as in Figure 1.

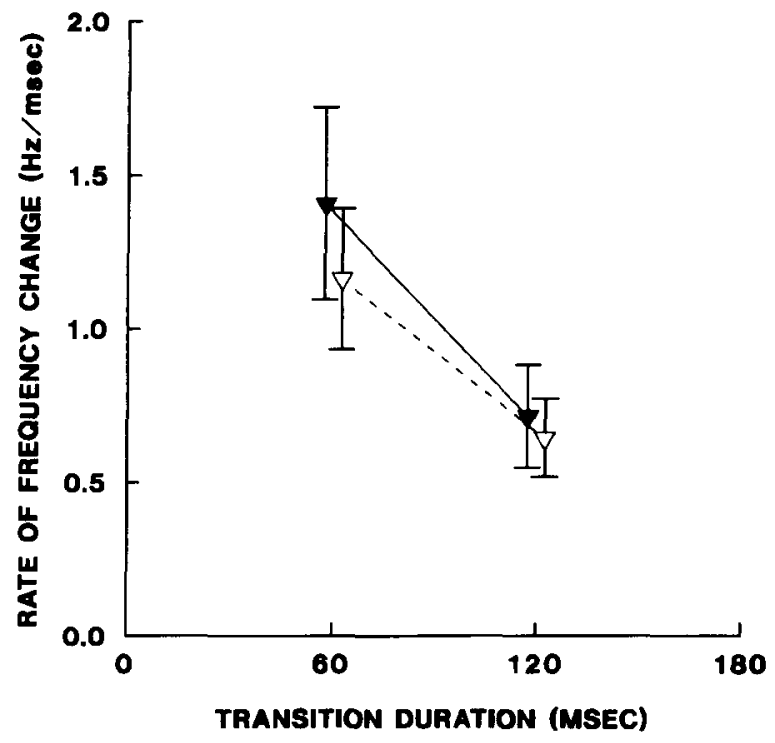

Figure 4. Mean 70\% correct values in hertz/millisecond for falling transitions, Experiment 2. Symbols: $\nabla$, DSO; $\nabla$, DSF. 
and repetition of hypotheses below). Results were very similar for both rising and falling transitions $[F(1,3)=$ $1.56, p>.3$ ], as predicted by Hypothesis 3 . These outcomes also confirmed results of Schouten and Pols's (1989) work with complex stimuli. Although the duration $\times$ direction interaction attained significance $[F(1,3)=11.58$, $p<.05$ ], it was uninterpretable.

\section{DISCUSSION}

The hypotheses that these experiments addressed will be reviewed: (1) Better discrimination should occur for transitions that diverge from a common frequency than for transitions that converge; (2) among sets of transitions that change at the same rate, discrimination of longer transitions should be better than discrimination of shorter transitions because the longer stimuli sweep through larger frequency differences; and (3) there should be no discrimination differences between rising and falling transitions if both sweep through the same frequency extents.

Better discrimination occurred among sets of transitions that diverged from a common frequency than among sets that converged. This occurred in both experiments and supported the pitch-extraction mechanism associated with Hypothesis 1. Furthermore, the significant differences between XSO and XSF stimuli, in both experiments, meant that subjects were not attending exclusively to the last $30 \mathrm{msec}$ of the stimuli. These last $30 \mathrm{msec}$ were identical for all members of a set. Had listeners attended only to these final portions of the stimuli, there should have been no differences between diverging and converging stimuli. Furthermore, within-set discrimination should not have been possible.

Better discrimination occurred for longer stimuli, supporting Hypothesis 2, and there were no differences between rising and falling stimuli, supporting Hypothesis 3. Both of the latter outcomes confirmed Schouten and Pols's (1989) conclusion that the "difference between initial and final frequencies of a sweep" are important for transition discrimination.

Table 2

Differences, in Hertz, Required for 50\% Correct Discrimination Between Transition 1 and Other Members of Each Stimulus Set

\begin{tabular}{|c|c|c|c|c|}
\hline \multirow{3}{*}{$\begin{array}{c}\text { Stimulus } \\
\text { Type }\end{array}$} & \multicolumn{4}{|c|}{ Transition Duration (msec) } \\
\hline & \multicolumn{2}{|c|}{60} & \multicolumn{2}{|c|}{120} \\
\hline & $M$ & $S D$ & $M$ & $S D$ \\
\hline \multicolumn{5}{|c|}{ Experiment 1} \\
\hline USO & 42.5 & 10.5 & 47.0 & 18.1 \\
\hline USF & 68.5 & 22.5 & 61.3 & 31.8 \\
\hline \multicolumn{5}{|c|}{ Experiment 2} \\
\hline USO & 25.2 & 8.9 & 23.4 & 7.9 \\
\hline USF & 39.6 & 11.3 & 32.4 & 14.9 \\
\hline DSO & 27.3 & 9.5 & 18.6 & 10.6 \\
\hline DSF & 37.8 & 10.0 & 36.0 & 6.2 \\
\hline
\end{tabular}

Note-Experiment 1 data were based on the adaptive JND procedure: Experiment 2 data were extracted from psychometric functions. Means and standard deviations for same onset (XSO) and same offset (XSF) stimuli. $X=U$ for rising transitions; $X=D$ for falling transitions.
Table 3

Differences, in Hertz, Required for $70 \%$ Correct Discrimination Between Transition 1 and Other Members of Each Stimulus Set, Experiment 2

\begin{tabular}{lccccc}
\hline & \multicolumn{4}{c}{ Transition Duration (msec) } \\
\cline { 2 - 3 } \cline { 5 - 6 } Stimulus & \multicolumn{3}{c}{60} & & \multicolumn{3}{c}{120} \\
\cline { 2 - 3 } \cline { 5 - 6 } Type & $M$ & $S D$ & & $M$ & \multicolumn{1}{c}{$S D$} \\
\hline USO & 58.5 & 14.3 & & 75.0 & 14.8 \\
USF & 78.3 & 14.0 & & 99.6 & 16.7 \\
DSO & 69.9 & 13.7 & & 78.0 & 15.2 \\
DSF & 84.6 & 18.7 & & 86.4 & 20.1 \\
\hline
\end{tabular}

Note-All data were extracted from the psychometric functions; otherwise, same as Table 2 .

Table 2 summarizes discrimination in terms of hertz differences at the $50 \%$ correct points for both experiments. If differences between initial and final frequencies of a "sweep" were the exclusive variable associated with transition discrimination, mean values in hertz should have been identical for each pair of 60 - and 120 -msec transitions. In five of the six instances of Table 2 , however, the values associated with 120 -msec transitions were numerically smaller than they were for 60 -msec stimuli (although some of the differences were small).

When one compares the data of Experiment 2 across Tables 2 and 3, all 50\% correct values were numerically smaller for 120 -msec transitions than for 60 -msec transitions. However, all $70 \%$ correct values were numerically larger for 120 -msec transitions than for the shorter stimuli. This suggests that the psychometric functions were slightly more shallow for the longer transitions than for $60-\mathrm{msec}$ stimuli. Why this occurred is not known with certainty. It may have happened because the change in transition duration altered energy characteristics of the stimuli or because it changed physiological processing mechanisms. The observed effect of differences in psychometric slopes for stimuli of different durations deserves further study and replication.

Results did not support the first of the two predictions associated with the excitation-pattern model, but did concur with the second-which, in turn, predicted the same outcome as Hypothesis 1. Results, therefore, were generally supportive of both pitch-extraction and sampling mechanisms for transition discrimination.

These outcomes suggest that, all other things being equal, discrimination of initial stop consonants that precede the same vowel should be poorer than discrimination of final consonants that follow the same vowel. This hypothesis is not in agreement with data of House, Williams, Hecker, and Kryter (1965), which showed better performance for initial natural-speech consonants than for final natural-speech consonants. However, this hypothesis does concur with Lacerda's (1987) data for discrimination within continua of $/ \mathrm{ba} /, / \mathrm{da} /, / \mathrm{ab} /$, and $/ \mathrm{ad} /$ synthetic stimuli in which only F2 varied. Lacerda found that better performance occurred for the latter two continua. The F2 transitions of those continua (i.e., /ab/and /ad/) might be considered as diverging from the same $F 2$ of the vowel of each continuum. 
The present study examined the perception of sounds similar to F2 transitions in stop consonants. Further investigations are needed to establish the mechanism(s) for the poorer discrimination between transitions that converge to the same frequency than between transitions that diverge. Systematic investigations of transition discrimination, vowel-consonant discrimination, and consonantvowel discrimination, by the same listeners, are needed to sort through these issues. Only this approach will fully test the hypothesis that discrimination of consonants preceding the same vowel is poorer than discrimination of consonants that follow the same vowel. Also, only this type of systematic approach will relate discrimination of synthesized transitions to perception of the complex, natural-speech signal.

\section{REFERENCES}

Arlinger, S. D., Jerlyall, L. B., Ahren, T., \& Holmgren, E. C. (1977). Thresholds for linear frequency ramps of a continuous pure tone. Acta Otolaryngologica, 83, 317-327.

Carlyon, R. P., StubBs, R. J. (1989). Detecting single-cycle frequency modulation imposed on sinusoidal, harmonic, and inharmonic carriers. Joumal of the Acoustical Society of America, 85, 2563-2574.

Coluns, M. J. (1984). Tone-glide discrimination of normal and hearingimpaired listeners. Joumal of Speech \& Hearing Research, 27, 403-412.

Collins, M. J., Cullen, J. K., Porter, R. J., \& Jackson, D. F. (1988). More support for rate-based discrimination of second formant transitions. Joumal of the Acoustical Society of America, 83(Suppl. 1), S83-S84.

DOOLEY, G. J., \& MoORE, B. C. J. (1988). Detection of linear frequency glides as a function of frequency and duration. Journal of the Acoustical Society of America, 84, 2045-2057.

Elliott, L. L., Hammer, M. A., Scholl, M. E., Carrell, T. D., \& WAsowicz, J. M. (1989). Discrimination of rising and falling simulated single-formant frequency transitions: Practice and duration effects. Journal of the Acoustical Society of America, 86, 945-953.

GARDNER, R. B., \& WILSON, J. P. (1979). Evidence for direction-specific channels in the processing of frequency modulation. Journal of the Acoustical Society of America, 66, 704-709.

House, A. S., Williams, C. E., Hecker, M. H. L., \& Kryter, K. D. (1965). Articulation-testing methods: Consonantal differentiation with a closed-response set. Joumal of the Acoustical Society of America, 37, $158-166$.

KLATT, D. H. (1980). Software for a cascade/parallel formant synthesizer. Joumal of the Acoustical Society of America, 67, 971-995.

KLATT, D. H. (1987). Speech-VAX Manual (Unpublished Tech. Rep. KLSYN). Cambridge: Massachusetts Institute of Technology.

LACERDA, F. (1987). Effects of stimulus dynamics on frequency discrimination. In M. E. H. Schouten (Ed.), The psychophysics of speech perception (pp. 250-257). Dordrecht, The Netherlands: Nijhoff.

LEVITT, H. (1971). Transformed up-down methods in psychoacoustics. Joumal of the Acoustical Society of America, 49, 467-477.
MoOre, B. C. J., \& Glasberg, B. R. (1989). Mechanisms underlying the frequency discrimination of pulsed tones and the detection of frequency modulation. Joumal of the Acoustical Society of America, 86, $1722-1732$.

NABELEK, I., \&IRSH, I. J. (1969). On the discrimination of frequency transitions. Journal of the Acoustical Society of America, 45, 1510-1519.

NabeleK, I. V., Nabelek, A. K., \& HirSh, I. J. (1970). Pitch of tone bursts of changing frequency. Joumal of the Acoustical Society of America, 48, 536-553.

POLLACK, I. (1968). Detection of rate of change of auditory frequency. Journal of Experimental Psychology, 77, 535-541.

Pols, L. C. W., \& Schouten, M. E. H. (1987). Perception of tone, band, and formant sweeps. In M. E. H. Schouten (Ed.), The psychophysics of speech perception (pp. 231-240). Dordrecht, The Netherlands: Nijhoff.

SCHOUTEN, M. E. H. (1985). Identification and discrimination of sweep tones. Perception \& Psychophysics, 37, 369-376.

Schouten, M. E. H., \& Pols, L. C. W. (1989). Identification and discrimination of sweep formants. Perception \& Psychophysics, 46, 235-244.

SERGEANT, R. L., \& HARRIS, J. D. (1962). Sensitivity to unidirectional frequency modulation. Joumal of the Acoustical Society of America, $34,1625-1628$.

Tyler, R. S., Wood, E. J., \& Fernandes, M. (1983). Frequency resolution and discrimination of constant and dynamic tones in normal and hearing-impaired listeners. Journal of the Acoustical Society of America, 74, 1190-1199.

ZWICKER, E. (1970). Masking and psychological excitation as consequences of the ear's frequency analysis. In R. Plomp \& G. F. Smoorenburg (Eds.), Frequency analysis and periodicity detection in hearing (pp. 376-394). Leiden, The Netherlands: Sijthoff.

\section{NOTES}

1. Experiment 1 was conducted during the summer of 1989 . Work by Schouten and Pols (1989), which concluded that "a pitch extraction mechanism cannot be the whole story" (p. 241) and which would argue against Hypothesis 1, had not yet been published.

2. The possible role of small amplitude differences (e.g., 1-3 dB) between different members of a stimulus set or within the duration of a stimulus was discussed by Elliott et al. (1989), who concluded that the small differences present in their stimuli did not affect transition discrimination. Nevertheless, this characteristic of stimuli produced with the Klatt (1987) synthesis software was controlled in developing stimuli employed in this study.

3. The last value had been intended to be the same as the preceding one, so that final-noise portions represented the approximate midpoints of transitions. The error was not discovered until testing was complete. However, the task always required discrimination within a set, all members of which shared the same noise. Also, the difference in final-noise center frequencies for USF and USO stimuli appeared to have no influence on results.

(Manuscript received October 30, 1990; revision accepted for publication February 28, 1991.) 